\title{
Theory of the Dielectric Susceptibility of Liquid Crystals with Polar Nonuniaxial Molecules
}

\author{
A. Kapanowski And T. Wietecha \\ Institute of Physics, Jagiellonian University \\ Reymonta 4, 30-059 Cracow, Poland
}

(Received May 21, 2004)

\begin{abstract}
Statistical theory of the dielectric susceptibility of polar liquid crystals is proposed. The molecules are not uniaxial but similar to cones. It is assumed that the permanent dipole moment of a molecule is parallel to the axis of the rotational symmetry. The ordering of the phase is described by means of the mean field theory based on the Maier-Saupe approach. The theory was used to calculate the temperature dependence of the order parameters and the susceptibilities. Predictions of the model for different sets of parameters are investigated.
\end{abstract}

PACS numbers: $61.30 . \mathrm{Cz}, 77.84 . \mathrm{Nh}$

\section{Introduction}

Liquid crystal phases consist of anisotropic molecules, either elongated or disc-like [1]. In many theoretical $[2,3]$ and computer simulation studies $[4,5]$ it is assumed that the molecules have the $D_{\infty h}$ (or $D_{2 h}$ ) symmetry and that the interactions do not change if a molecule will point to the opposite direction. However, real molecules are always less symmetrical. Somoza and Tarazona [6] showed that the molecular asymmetry can influence the packing fraction, the relative density jump and the order parameter at the isotropic-nematic transition for hard body systems. Recently Barmes et al. [7] reported results from Monte Carlo simulations investigating mesophase formation in systems of hard pear-shaped particles. For different elongations isotropic, nematic, interdigitated smectic $A_{2}$ phases were obtained and sometimes glassy behaviour was seen. Pear-shaped molecules were also used by Davidson and Mottram [8] in a continuum theory model of switching in a bistable nematic liquid crystal device. 
In the molecular-statistical theory of Maier and Saupe the potential energy of interactions is

$$
V\left(\boldsymbol{n}_{1}, \boldsymbol{n}_{2}\right)=v_{0}+v_{2} P_{2}\left(\boldsymbol{n}_{1} \cdot \boldsymbol{n}_{2}\right),
$$

where $P_{j}$ are the Legendre polynomials, the vectors $\boldsymbol{n}_{1}, \boldsymbol{n}_{2}$ determine the orientation of molecules. There is a nematic phase for $v_{2}<0$ ( $v_{0}$ is not important). We would like to investigate the interactions that depend on the sense of the vectors $\boldsymbol{n}_{1}, \boldsymbol{n}_{2}$ (it corresponds to the $C_{\infty v}$ symmetry of molecules)

$$
V\left(\boldsymbol{n}_{1}, \boldsymbol{n}_{2}\right)=v_{0}+v_{1} P_{1}\left(\boldsymbol{n}_{1} \cdot \boldsymbol{n}_{2}\right)+v_{2} P_{2}\left(\boldsymbol{n}_{1} \cdot \boldsymbol{n}_{2}\right) .
$$

The parameters $v_{i}$ can be chosen by means of the excluded volume method described by Straley [9]. As a result we get always $v_{1} \geq 0$. The energy of interactions will be lower for $\boldsymbol{n}_{1}=-\boldsymbol{n}_{2}$ and this leads to the conclusion that only isotropic and nematic phases can be obtained.

We can obtain negative (or positive) $v_{1}$ and new ordering of the phase if we consider interactions of two electric dipoles. Under the assumption that $\boldsymbol{p}=p \boldsymbol{n}$, the interactions have the form

$$
U_{12}\left(\boldsymbol{u}, \boldsymbol{n}_{1}, \boldsymbol{n}_{2}\right)=\frac{p^{2}\left[\left(\boldsymbol{n}_{1} \cdot \boldsymbol{n}_{2}\right)-3\left(\boldsymbol{n}_{1} \cdot \boldsymbol{\Delta}\right)\left(\boldsymbol{n}_{2} \cdot \boldsymbol{\Delta}\right)\right]}{4 \pi \epsilon_{0} u^{3}},
$$

where $\boldsymbol{u}=u \boldsymbol{\Delta}$ is the distance between dipoles. We would like to get average interactions that do not depend on the vector $\boldsymbol{\Delta}$ :

$$
U_{12}^{\mathrm{av}}\left(u, \boldsymbol{n}_{1}, \boldsymbol{n}_{2}\right)=\frac{1}{4 \pi} \int \mathrm{d} \boldsymbol{\Delta} U_{12}\left(\boldsymbol{u}, \boldsymbol{n}_{1}, \boldsymbol{n}_{2}\right) g\left(\boldsymbol{\Delta}, \boldsymbol{n}_{1}, \boldsymbol{n}_{2}\right),
$$

where the function $g\left(\boldsymbol{\Delta}, \boldsymbol{n}_{1}, \boldsymbol{n}_{2}\right)$ describes the neighbourhood of the molecule 1 (how often we can find the molecule 2 in the direction $\boldsymbol{\Delta}$ at the constant $u$ ). The normalization is

$$
\frac{1}{4 \pi} \int \mathrm{d} \boldsymbol{\Delta} g\left(\boldsymbol{\Delta}, \boldsymbol{n}_{1}, \boldsymbol{n}_{2}\right)=1
$$

For isotropic distribution (spherical molecules) $g\left(\boldsymbol{\Delta}, \boldsymbol{n}_{1}, \boldsymbol{n}_{2}\right)=1$ and we get

$$
U_{12}^{\text {av }}\left(\boldsymbol{n}_{1}, \boldsymbol{n}_{2}\right)=0 \text {. }
$$

But for anisotropic molecules it is more reasonable to try different form of the function

$$
g\left(\boldsymbol{\Delta}, \boldsymbol{n}_{1}, \boldsymbol{n}_{2}\right)=1-a P_{2}\left(\boldsymbol{n}_{1} \cdot \boldsymbol{\Delta}\right)-a P_{2}\left(\boldsymbol{n}_{2} \cdot \boldsymbol{\Delta}\right)
$$

where $a$ is related to the anisotropy of molecules: rod-like (disc-like) molecules have $a>0(a<0)$ :

$$
U_{12}^{\mathrm{av}}\left(\boldsymbol{n}_{1}, \boldsymbol{n}_{2}\right)=\frac{p^{2}(4 / 5) a\left(\boldsymbol{n}_{1} \cdot \boldsymbol{n}_{2}\right)}{4 \pi \epsilon_{0} u^{3}} .
$$

Thus, for disc-like molecules $(a<0)$ one can use $v_{1}<0$ and this will produce ferroelectric ordering for sufficiently low temperatures. Generally, we can 
say that $v_{1}$ describes the result of sum of the excluded volume effects and dipolar interactions. Our aim is to investigate the influence of the $v_{1}$ term on the ordering and the dielectric susceptibility of the phase.

As far as the susceptibility is concerned, we would like to recall some results in order to provide the context for our considerations. The potential energy of the dipole $\boldsymbol{p}$ in the electric field $\boldsymbol{E}$ is $U=-\boldsymbol{p} \cdot \boldsymbol{E}$. For a system of $N$ noninteracting dipole moments in the volume $V$ we get the polarization $P$ from the Boltzmann distribution

$$
P=\frac{N p}{V} L(\beta p E),
$$

where $\beta=1 / k_{\mathrm{B}} T$ and $L(x)=\operatorname{coth} x-1 / x$ is the Langevin function. For small values of the argument, $L(x) \approx x / 3$, and thus

$$
P=\frac{N p^{2} \beta E}{3 V} \text {. }
$$

The susceptibility is defined as

$$
\epsilon_{0} \chi=\frac{\partial P}{\partial E}=\frac{N p^{2}}{3 V k_{\mathrm{B}} T} .
$$

We get a $1 / T$ dependence called the Curie law. It indicates that the dipoles are noninteracting.

For the anisotropic polar molecules and anisotropic phases the susceptibility is a tensor

$$
\epsilon_{0} \chi_{\alpha \beta}=\frac{\partial P_{\alpha}}{\partial E_{\beta}} .
$$

The polarization can be written as

$$
P_{\alpha}=\frac{N p}{V}\left\langle\bar{n}_{\alpha}\right\rangle
$$

where $p\left\langle\bar{n}_{\alpha}\right\rangle$ is the average value of the dipole component in the $\alpha$-direction in the presence of the electric field. Hence two different averages are involved. The bar refers to the potential energy of the dipole moment in the electric field and the brackets to the nematic potential. We calculate it using the linear approximation

$$
\left\langle\bar{n}_{\alpha}\right\rangle=\left\langle n_{\alpha}\left(1+\beta p n_{\beta} E_{\beta}\right)\right\rangle=\beta p\left\langle n_{\alpha} n_{\beta}\right\rangle E_{\beta} .
$$

The susceptibility is

$$
\epsilon_{0} \chi_{\alpha \beta}=\frac{N p^{2} \beta}{V}\left\langle n_{\alpha} n_{\beta}\right\rangle .
$$

The components of the susceptibility in the nematic phase oriented along the $z$ axis are

$$
\epsilon_{0} \chi_{z z}=\frac{N p^{2}(1+2 S)}{3 V k_{\mathrm{B}} T},
$$




$$
\epsilon_{0} \chi_{x x}=\frac{N p^{2}(1-S)}{3 V k_{\mathrm{B}} T}
$$

where $S=\left\langle P_{2}\left(n_{z}\right)\right\rangle$ is the order parameter. Let us note that the average susceptibility is

$$
\bar{\chi} \equiv \frac{1}{3}\left(\chi_{z z}+2 \chi_{x x}\right)=\frac{N p^{2} \beta}{3 V \epsilon_{0}}=\chi^{\text {iso }}
$$

as for the isotropic phase. The equality $\bar{\chi}=\chi^{\text {iso }}$ is correct for nonpolar molecules but for polar ones we know from the experiments that $\bar{\chi}<\chi^{\text {iso }}$. More advanced calculations were given by Maier and Meier [10] who extended the Onsager theory of the susceptibility to nematic liquid crystals. If we neglect the induced polarization we can write their results as

$$
\begin{aligned}
& \epsilon_{0} \chi_{z z}=\left(\frac{3 \tilde{\chi}+3}{2 \tilde{\chi}+3}\right) \frac{N p^{2}(1+2 S)}{3 V k_{\mathrm{B}} T}, \\
& \epsilon_{0} \chi_{x x}=\left(\frac{3 \tilde{\chi}+3}{2 \tilde{\chi}+3}\right) \frac{N p^{2}(1-S)}{3 V k_{\mathrm{B}} T},
\end{aligned}
$$

where $\tilde{\chi}$ is the average susceptibility of the phase.

The organization of this paper is as follows: in Sec. 2 the mean field theory of the nematic ordering is provided. In Sec. 3 the formulae for the susceptibility are derived. Section 4 is devoted to some applications of the presented theory. Section 5 contains a summary. Appendixes provide the definitions and main properties of the basic functions (Appendix A) and the excluded volumes for molecules of different shape (Appendix B).

\section{The mean field theory}

The potential energy of molecular interactions of the molecules is given by

$$
V\left(R_{1}, R_{2}\right)=\sum_{j=0,1,2} v_{j} E_{00}^{(j)}\left(R_{2}^{-1} R_{1}\right)
$$

where $R_{i}$ are the sets of the three Euler angles describing the orientation of the molecule $i, E_{\mu \nu}^{(j)}$ are the basic functions defined in Appendix A. The molecular orientation in the phase is described by the distribution function

$$
\int \mathrm{d} R f(R)=1
$$

We calculate the mean of some function $A=A(R)$ as

$$
\langle A\rangle \equiv \int \mathrm{d} R f(R) A(R)
$$

The state of the system is described by order parameters $\left\langle E_{\mu 0}^{(j)}\right\rangle$ but the most important is $S=\left\langle E_{00}^{(2)}\right\rangle$. The internal energy of the system is 


$$
U=\frac{N}{2} \int \mathrm{d} R_{1} \mathrm{~d} R_{2} f\left(R_{1}\right) f\left(R_{2}\right) V\left(R_{1}, R_{2}\right),
$$

whereas the entropy of the system is written as

$$
S=-k_{\mathrm{B}} N \int \mathrm{d} R f(R) \ln \left[f(R) C_{N}\right] .
$$

The energy of permanent dipole moments $\boldsymbol{p}=p \boldsymbol{n}$ in the internal electric field $\boldsymbol{E}^{\text {int }}$ is

$$
U^{E}=N\left\langle\boldsymbol{p} \cdot \boldsymbol{E}^{\text {int }}\right\rangle
$$

Generally, the internal electric field differs from the external field $\boldsymbol{E}^{\text {ext }}$ [11]. Here we assume that both fields are equal $\boldsymbol{E}^{\text {int }}=\boldsymbol{E}^{\text {ext }}=\boldsymbol{E}$.

The total free energy of the system is the sum

$$
F^{\text {tot }}=U+U^{E}-T S \text {. }
$$

In the mean-field approximation we get a potential energy

$$
W(R)=\sum_{j} \sum_{\mu} w_{\mu 0}^{(j)} E_{\mu 0}^{(j)}(R),
$$

and from the Boltzmann distribution we get

$$
f(R)=\exp [-\beta W(R)] / Z,
$$

where $Z$ is a normalization constant. The consistency condition

$$
W\left(R_{1}\right)=\int \mathrm{d} R_{2} f\left(R_{2}\right) V\left(R_{1}, R_{2}\right)-\boldsymbol{p}\left(R_{1}\right) \cdot \boldsymbol{E}
$$

leads to equations

$$
w_{\mu 0}^{(j)}=\left\langle E_{\mu 0}^{(j)}\right\rangle v_{j}-p \delta_{1 j}\left(E_{x} \delta_{1 \mu}-E_{y} \delta_{-1 \mu}+E_{z} \delta_{0 \mu}\right) .
$$

It is useful to introduce the dimensionless parameters $S_{\mu 0}^{(j)}=-\beta w_{\mu 0}^{(j)}$ for $j>0$ :

$$
\ln f(R)=\sum_{j} \sum_{\mu} S_{\mu 0}^{(j)} E_{\mu 0}^{(j)}(R) .
$$

$S_{00}^{(0)}$ is responsible for the normalization and it depends on other $S_{\mu 0}^{(j)}$ with $j>0$ :

$$
\begin{aligned}
& S_{00}^{(0)}=-\ln \left[\int \mathrm{d} R \exp \left(\sum_{j>0} \sum_{\mu} S_{\mu 0}^{(j)} E_{\mu 0}^{(j)}(R)\right)\right], \\
& \frac{\partial S_{00}^{(0)}}{\partial S_{\mu 0}^{(j)}}=-\left\langle E_{\mu 0}^{(j)}\right\rangle \\
& W_{\mu \nu}^{j k} \equiv \frac{\partial\left\langle E_{\mu 0}^{(j)}\right\rangle}{\partial S_{\nu 0}^{(k)}}=\left\langle E_{\mu 0}^{(j)} E_{\nu 0}^{(k)}\right\rangle-\left\langle E_{\mu 0}^{(j)}\right\rangle\left\langle E_{\nu 0}^{(k)}\right\rangle .
\end{aligned}
$$


The equations have the form

$$
S_{\mu 0}^{(j)}+\beta v_{j}\left\langle E_{\mu 0}^{(j)}\right\rangle=\beta p \delta_{1 j}\left(E_{x} \delta_{1 \mu}-E_{y} \delta_{-1 \mu}+E_{z} \delta_{0 \mu}\right) .
$$

The solution is orientationally stable only if the matrix

$$
\left[W_{\mu \nu}^{j k}+\sum_{l>0} \sum_{\rho} \beta v_{l} W_{\mu \rho}^{j l} W_{\nu \rho}^{k l}\right]
$$

is positive definite. The isotropic phase is orientationally stable if $\beta v_{1}>-3$ and $\beta v_{2}>-5$.

\section{The dielectric susceptibility}

The dielectric susceptibility tensor is defined by Eq. (12) and we calculate the orientational polarization as

$$
P_{\alpha}=\left\langle n_{\alpha}\right\rangle N p / V
$$

Let us note that the polarization depends on the electric field via the distribution function. The components of the susceptibility are

$$
\begin{aligned}
& \epsilon_{0} \chi_{z z}=\frac{N p}{V} \sum_{j>0} \sum_{\mu} W_{0 \mu}^{1 j} \frac{\partial S_{\mu 0}^{(j)}}{\partial E_{z}}, \\
& \epsilon_{0} \chi_{x x}=\frac{N p}{V} \sum_{j>0} \sum_{\mu} W_{1 \mu}^{1 j} \frac{\partial S_{\mu 0}^{(j)}}{\partial E_{x}},
\end{aligned}
$$

where the derivatives are calculated from the equations

$$
\begin{aligned}
& \sum_{k>0} \sum_{\nu}\left(\delta_{j k} \delta_{\mu \nu}+\beta v_{j} W_{\mu \nu}^{j k}\right) \frac{\partial S_{\nu 0}^{(k)}}{\partial E_{z}}=\beta p \delta_{1 j} \delta_{0 \mu}, \\
& \sum_{k>0} \sum_{\nu}\left(\delta_{j k} \delta_{\mu \nu}+\beta v_{j} W_{\mu \nu}^{j k}\right) \frac{\partial S_{\nu 0}^{(k)}}{\partial E_{x}}=\beta p \delta_{1 j} \delta_{1 \mu} .
\end{aligned}
$$

Now we are in the position to discuss the results for different possible phases.

\subsection{The isotropic phase}

For the zero field all order parameters are equal to zero. For the nonzero field the phase has the symmetry $C_{\infty v}$ (the symmetry of the electric field). Thus we have to use the parameters $S_{00}^{(j)}$ only.

$$
\epsilon_{0}^{\text {iso }}=\epsilon_{0} \chi_{z z}=\epsilon_{0} \chi_{x x}=\frac{N p^{2}}{V\left(3 k_{\mathrm{B}} T+v_{1}\right)} .
$$

For $v_{1}<0$ we get the Curie-Weiss law describing the divergence of $\chi$ when we approach the Curie temperature from above. For $v_{1}>0$ the susceptibility is finite. 


\subsection{The uniaxial nematic phase}

For the zero field the phase has the symmetry $D_{\infty h}$ and the parameters $S_{00}^{(j)}$ with $j$ even are present. For the parallel field the phase has the symmetry $C_{\infty v}$ and the parameters $S_{00}^{(j)}$ with all $j$ are present. For the perpendicular field the phase has the symmetry $C_{2 v}$ and we have the parameters $S_{00}^{(j)}$ with $j$ even and $S_{\mu 0}^{(j)}$ with $j, \mu$ odd. The expressions $W_{\mu \nu}^{j k}$ with $j+k$ and $\mu+\nu$ even are nonzero.

$$
\begin{aligned}
\epsilon_{0} \chi_{z z} & =\frac{N p^{2} \beta W_{00}^{11}}{V\left(1+\beta v_{1} W_{00}^{11}\right)}, \\
\epsilon_{0} \chi_{x x} & =\frac{N p^{2} \beta W_{11}^{11}}{V\left(1+\beta v_{1} W_{11}^{11}\right)} .
\end{aligned}
$$

Let us note that for $v_{1}>0$ we get $\bar{\chi}<\chi^{i s o}$.

\subsection{The ferroelectric phase}

For the zero field and for the parallel field the phase has the symmetry $C_{\infty v}$ and the parameters $S_{00}^{(j)}$ with all $j$ are present. The expressions $W_{\mu \nu}^{j k}$ with $\mu+\nu$ even are nonzero.

$$
\epsilon_{0} \chi_{z z}=\left(\frac{N p^{2} \beta}{V}\right) \frac{W_{00}^{11}\left(1+\beta v_{2} W_{00}^{22}\right)-\beta v_{2}\left(W_{00}^{12}\right)^{2}}{\left(1+\beta v_{1} W_{00}^{11}\right)\left(1+\beta v_{2} W_{00}^{22}\right)-\beta v_{1} \beta v_{2}\left(W_{00}^{12}\right)^{2}} .
$$

For convenience, we express the important elements $W_{\mu \nu}^{j k}$ by means of the order parameters $\left\langle P_{j}\right\rangle$ in the case of the ferroelectric phase

$$
\begin{aligned}
& W_{00}^{11}=\left(1+2\left\langle P_{2}\right\rangle\right) / 3-\left\langle P_{1}\right\rangle^{2}, \\
& W_{00}^{12}=\left(2\left\langle P_{1}\right\rangle+3\left\langle P_{3}\right\rangle\right) / 5-\left\langle P_{1}\right\rangle\left\langle P_{2}\right\rangle, \\
& W_{00}^{22}=\left(7+10\left\langle P_{2}\right\rangle+18\left\langle P_{4}\right\rangle\right) / 35-\left\langle P_{2}\right\rangle^{2}, \\
& W_{11}^{11}=\left(1-\left\langle P_{2}\right\rangle\right) / 3, \\
& W_{11}^{12}=\left(\left\langle P_{1}\right\rangle-\left\langle P_{3}\right\rangle\right) \sqrt{3} / 5, \\
& W_{11}^{22}=\left(7+5\left\langle P_{2}\right\rangle-12\left\langle P_{4}\right\rangle\right) / 35, \\
& W_{20}^{22}=0, \\
& W_{22}^{22}=\left(7-10\left\langle P_{2}\right\rangle+3\left\langle P_{4}\right\rangle\right) / 35, \\
& W_{02}^{12}=0 .
\end{aligned}
$$

\section{Exemplary calculations}

In this section we carry out calculations for different physical systems of polar molecules described by the considered model. The phase diagram of the 


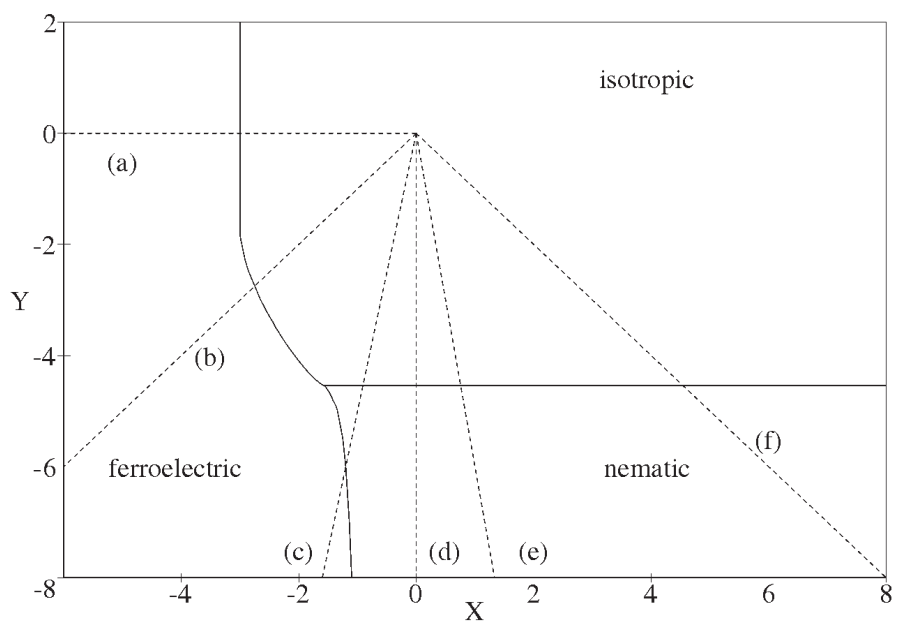

Fig. 1. Phase diagram of the model considered in the paper, where $X=\beta v_{1}$ and $Y=\beta v_{2}$. Three phases are present: isotropic, uniaxial nematic, and ferroelectric. Dashed half-lines denote different physical systems: $(a) v_{2}=0,(b) v_{2}=v_{1},(c) v_{2}=$ $5 v_{1},(d) v_{1}=0,(e) v_{2}=-6 v_{1}$, and $(f) v_{2}=-v_{1}$. For a given physical system on decreasing the temperature we are moving from the centre $(0,0)$ to the edge of the figure.

model is shown in Fig. 1. Three phases are present: isotropic, uniaxial nematic, and ferroelectric. Dashed half-lines in the picture denote different physical systems with the fixed parameters $v_{1}$ and $v_{2}$ (in fact, the fixed ratio $v_{2}$ to $v_{1}$ is important). The parameters of the model were calculated by means of the excluded volume method. The formulae for molecules with different shapes are given in Appendix B.

Let us start the description of the results from the system of uniaxial molecules similar to ellipsoids or cylinders [half-line $(d)$ in Fig. $1, v_{1}=0$ ]. In all pictures, $T$ denotes the dimensionless temperature. $T=1$ corresponds to the transition from the isotropic to the nematic or ferroelectric phase. The susceptibilities are scaled in order to obtain $\chi=1$ for $T=1$. The temperature dependence of the order parameter $\left\langle P_{2}\right\rangle$ and the susceptibilities are presented in Figs. 2 and 3, respectively. There is the first-order transition from the isotropic to the nematic phase. On decreasing the temperature the susceptibility splits into $\chi_{x x}<\chi_{z z} \cdot \chi_{z z}$ runs to the infinity whereas $\chi_{x x}$ remains finite. This is typical of the uniaxial molecules with the dipole moment parallel to the symmetry axis.

Let us consider less symmetrical molecules similar to cones with $H / D=4$, where $H$ and $D$ denote the height and the diameter of the base, respectively [half-line $(e)$ in Fig. 1, $v_{2}=-6 v_{1}$ ]. Let us note that only the isotropic and nematic phases are present. This reflects the fact that liquid crystals are in general nonpolar. On the other hand, one should expect the flexoelectric effect which is polar [12]. The temperature dependence of the order parameter $\left\langle P_{2}\right\rangle$ is similar to 


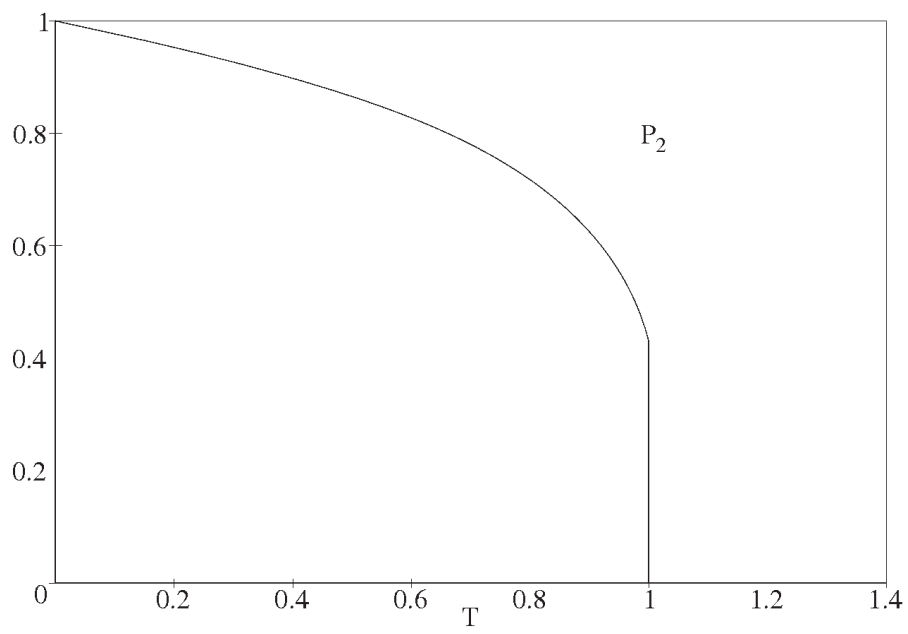

Fig. 2. Temperature dependence of the order parameter $\left\langle P_{2}\right\rangle$ for $v_{1}=0$ [half-line $(d)$ in Fig. 1]. There is the first-order transition from the isotropic to the uniaxial nematic phase. $T$ denotes the dimensionless temperature.

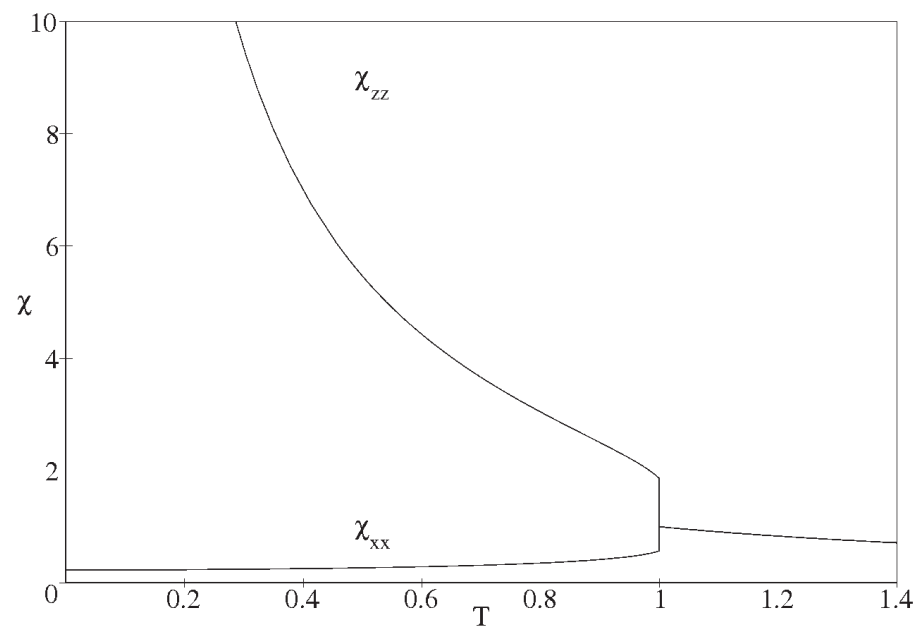

Fig. 3. Temperature dependence of the susceptibilities for $v_{1}=0$ [half-line $(d)$ in Fig. 1]. On decreasing the temperature the susceptibility splits into $\chi_{x x}<\chi_{z z}$. For $T \rightarrow 0, \chi_{z z}$ becomes infinite. $T$ denotes the dimensionless temperature.

shown in Fig. 2. The temperature dependence of the susceptibilities is presented in Fig. 4. We have known splitting of the susceptibility but here both $\chi_{x x}$ and $\chi_{z z}$ are finite. In Fig. 5 the results for $v_{2}=-v_{1}$ are given [half-line $(f)$ in Fig. 1] where $\chi_{x x}$ and $\chi_{z z}$ are almost the same order. Let us note that this value of the $v_{2} / v_{1}$ cannot be obtained from the excluded volume method but we use it in order to understand the behaviour of the model. We conclude that if cones become longer 


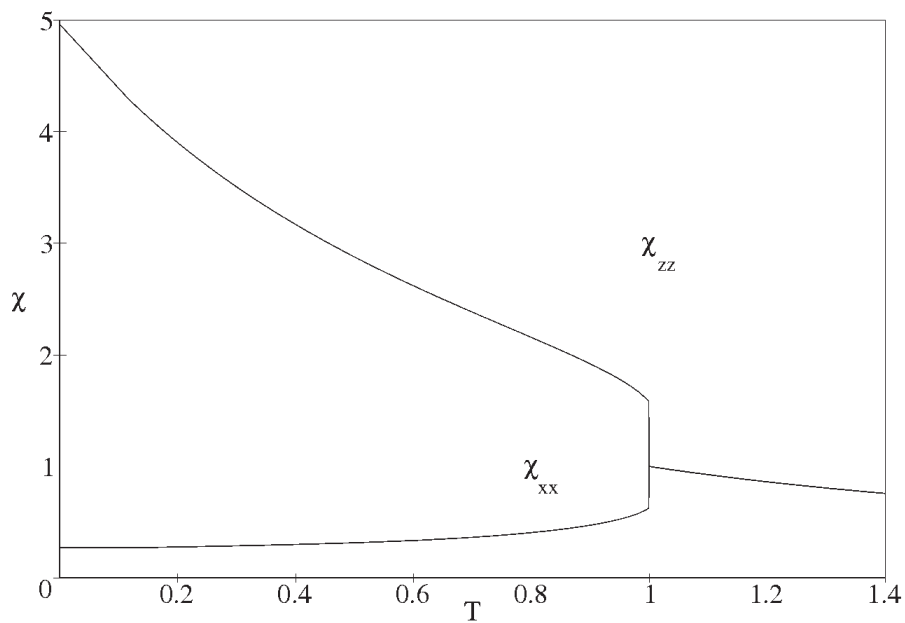

Fig. 4. Temperature dependence of the susceptibilities for $v_{2}=-6 v_{1}$ [half-line $(e)$ in Fig. 1]. Both susceptibilities are finite. $T$ denotes the dimensionless temperature.

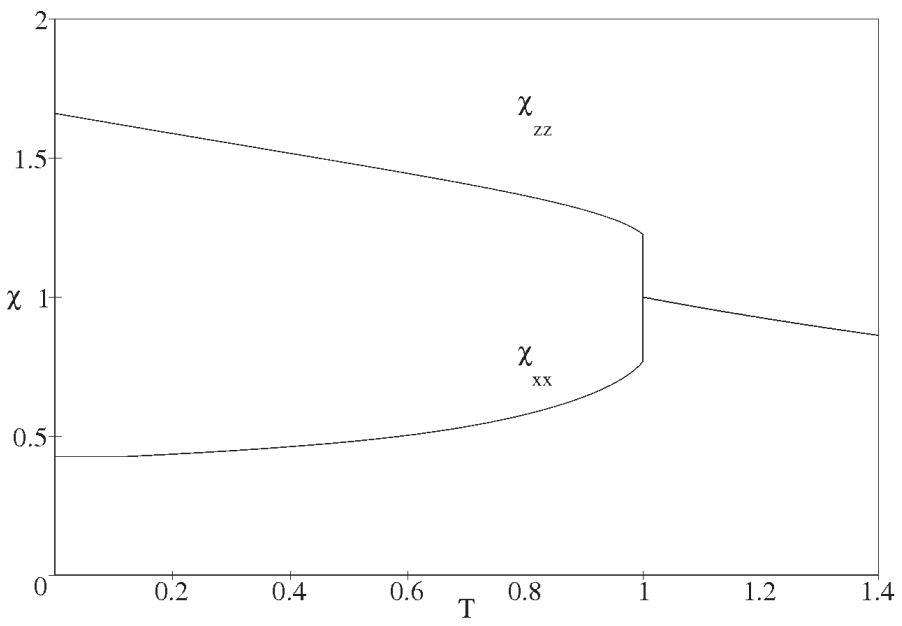

Fig. 5. Temperature dependence of the susceptibilities for $v_{2}=-v_{1}$ [half-line $(f)$ in Fig. 1]. This value of the $v_{2} / v_{1}$ cannot be obtained from the excluded volume method. $T$ denotes the dimensionless temperature.

then their properties are more similar to ellipsoids and cylinders. For shorter cones it is more difficult for the electric field to rotate the molecule and that is why the susceptibility $\chi_{z z}$ is lower (but still greater than $\chi_{x x}$ ).

A ferroelectric phase is present in the considered model when the parameter $v_{1}$ is negative and there is a resultant tendency to arrange the dipoles in a parallel fashion. The temperature dependence of the order parameters and the susceptibilities in the case of $v_{2}=5 v_{1}$ [half-line $(c)$ in Fig. 1] are presented in Figs. 6 and 7 , 


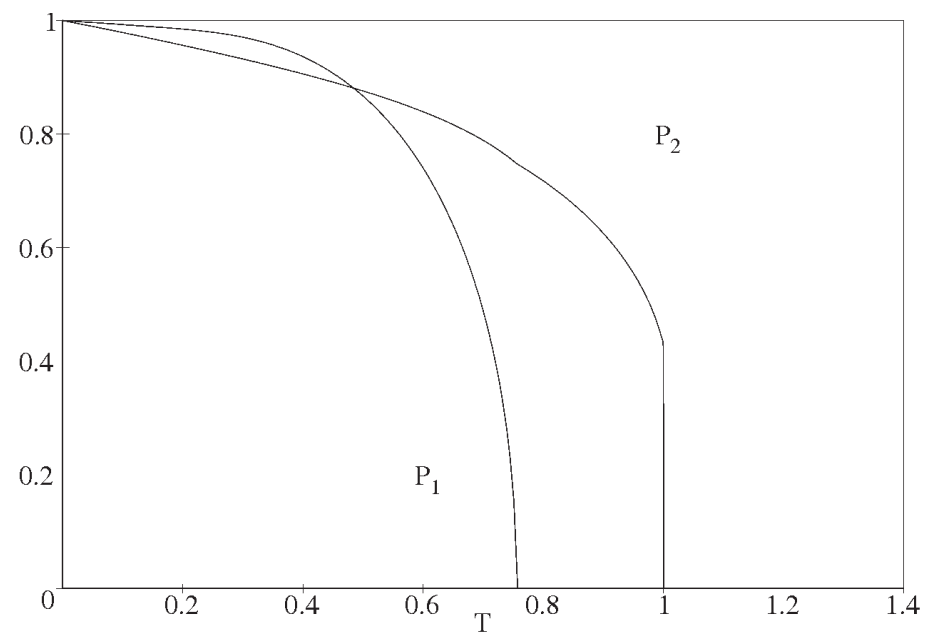

Fig. 6. Temperature dependence of the order parameter $\left\langle P_{1}\right\rangle$ and $\left\langle P_{2}\right\rangle$ for $v_{2}=5 v_{1}$ [half-line $(c)$ in Fig. 1]. There is the first-order transition from the isotropic to the uniaxial nematic phase at $T=1$ and next, the second-order transition to the ferroelectric phase at $T=0.763$. $T$ denotes the dimensionless temperature.

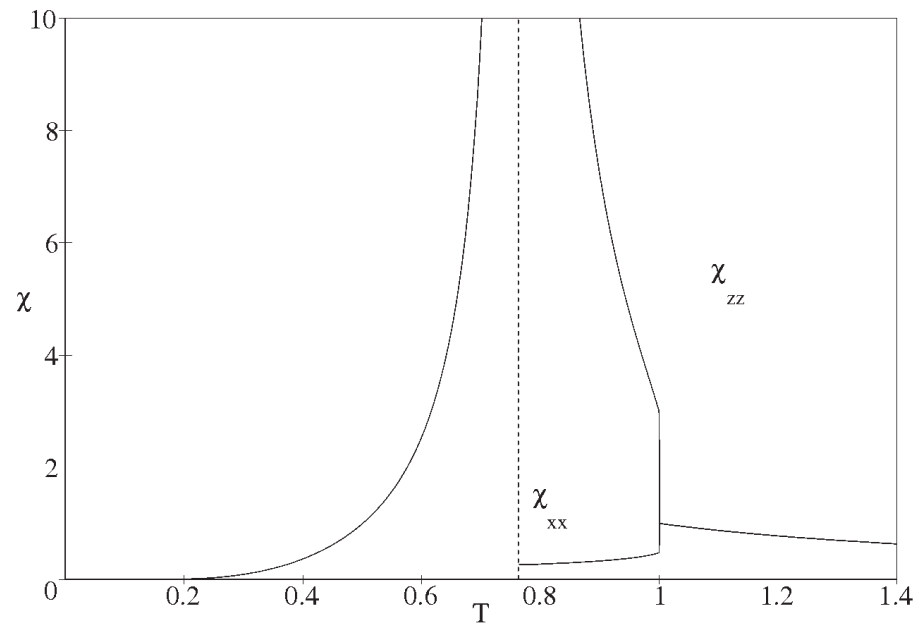

Fig. 7. Temperature dependence of the susceptibilities for $v_{2}=5 v_{1}$ [half-line $(c)$ in Fig. 1]. In the nematic phase we have $\chi_{x x}<\chi_{z z}$. At the transition from the nematic to the ferroelectric phase $\chi_{z z}$ diverges (the Curie-Weiss law). The dashed line denotes the transition point between nematic and ferroelectric phases. $T$ denotes the dimensionless temperature.

respectively. There is the first-order transition from the isotropic to the nematic phase at $T=1$. Next, there is the second-order transition to the ferroelectric phase at $T=0.763$. 


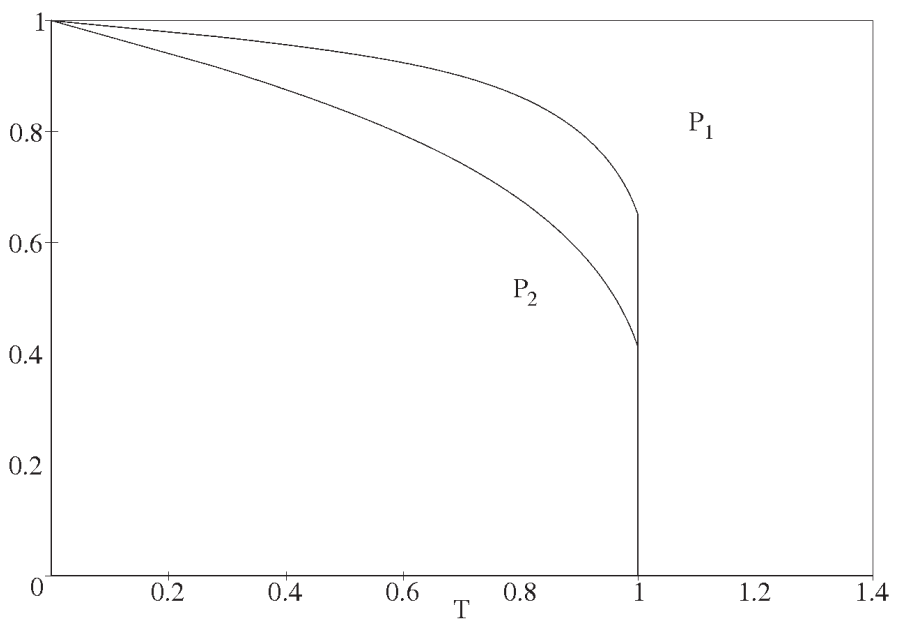

Fig. 8. Temperature dependence of the order parameters $\left\langle P_{1}\right\rangle$ and $\left\langle P_{2}\right\rangle$ for $v_{2}=v_{1}$ [half-line $(b)$ in Fig. 1]. There is the first-order transition from the isotropic to the ferroelectric phase. $T$ denotes the dimensionless temperature.

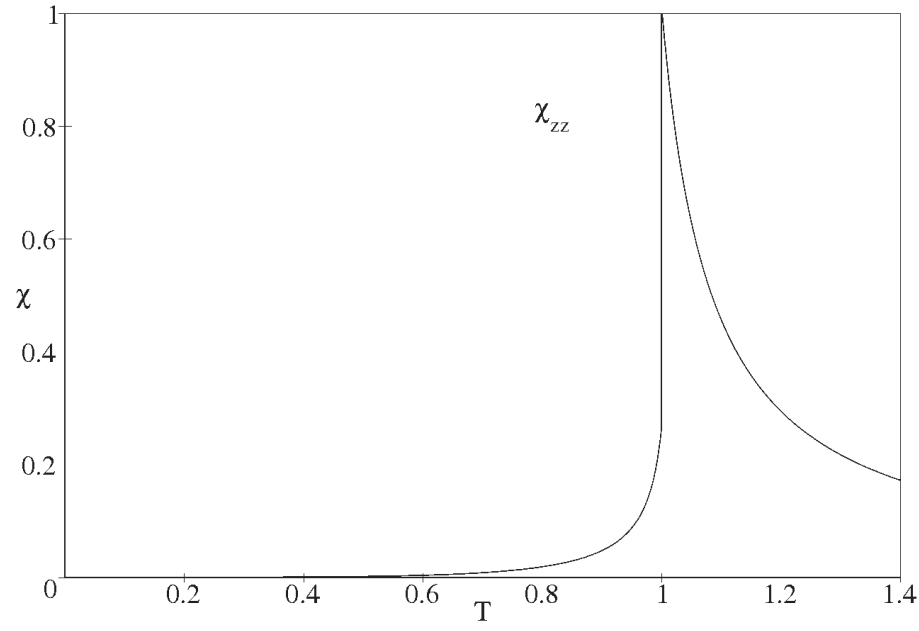

Fig. 9. Temperature dependence of the susceptibilities for $v_{2}=v_{1}$ [half-line $(b)$ in Fig. 1]. $\chi_{z z}$ is finite and it has the strong maximum at the transition from the isotropic to the ferroelectric phase. $T$ denotes the dimensionless temperature.

The temperature dependence of the order parameters and the susceptibilities in the case of $v_{2}=v_{1}$ [half-line $(b)$ in Fig. 1] are presented in Figs. 8 and 9, respectively. There is the first-order transition from the isotropic to the ferroelectric phase. $\chi_{z z}$ is always finite and it has the strong maximum at the transition.

For the case of $v_{2}=0$ we get the simple equation for $S_{00}^{(1)}$ only [half-line $(a)$ in Fig. 1] 


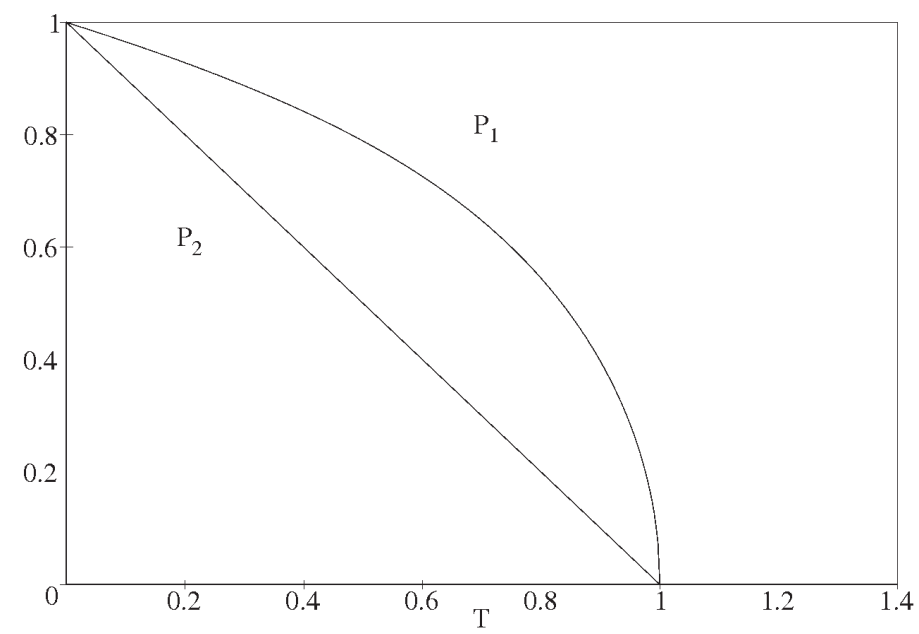

Fig. 10. Temperature dependence of the order parameters $\left\langle P_{1}\right\rangle$ and $\left\langle P_{2}\right\rangle$ for $v_{2}=0$ [half-line $(a)$ in Fig. 1]. There is the second-order transition from the isotropic to the ferroelectric phase. $T$ denotes the dimensionless temperature.

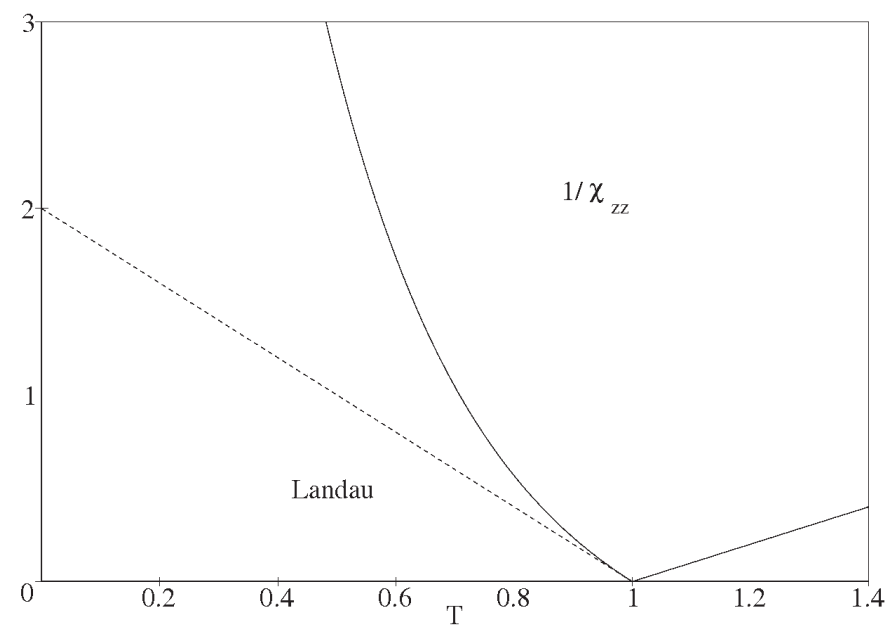

Fig. 11. Temperature dependence of the inverse of the susceptibilities for $v_{2}=0$ [half-line $(a)$ in Fig. 1]. The susceptibility $\chi_{z z}$ in the ferroelectric phase is half of that in the isotropic phase (in the neighbourhood of the transition point). The dashed line shows the results from the Landau description. $T$ denotes the dimensionless temperature.

$$
S_{00}^{(1)}+\beta v_{1} L\left(S_{00}^{(1)}\right)=\beta p E_{z} .
$$

In the zero field there is the second-order transition from the isotropic to the ferroelectric phase at $T_{\mathrm{C}}=-v_{1} / 3 k_{\mathrm{B}}$. The order parameters shown in Fig. $10 \mathrm{can}$ be expressed as 


$$
\begin{aligned}
& \left\langle P_{1}\right\rangle=L\left(S_{00}^{(1)}\right), \\
& \left\langle P_{2}\right\rangle=1-T / T_{\mathrm{C}} .
\end{aligned}
$$

As far as the susceptibility is concerned, we recover a general mean field result: the susceptibility in the ordered phase is half of that in the disordered phase (at the neighbourhood of the transition point) [13]. The reason is that the effect of an external field on the polarization must be smaller in the ordered phase, due to the existing internal field in that phase, which has a stabilizing effect on the polarization. In Fig. 11 we plot the inverse of the susceptibility as a function of the temperature. The dashed line shows the results from the Landau description for the ferroelectric phase. We have an expected agreement in the neighbourhood of the transition point.

\section{Summary}

In this paper we considered the mean field theory with interactions which are not uniaxial. We showed that they correspond to molecules similar to cones or molecules with permanent dipole moments parallel to the axis of rotational symmetry. Three different phases were obtained: isotropic (I), nematic (N), and ferroelectric $(\mathrm{F})$. We found the first- $(\mathrm{I}-\mathrm{N}, \mathrm{I}-\mathrm{F})$ and the second-order transitions $(\mathrm{N}-\mathrm{F}, \mathrm{I}-\mathrm{F})$ for some sets of parameters.

We also derived the formulae for the dielectric susceptibility in the case of polar molecules. We neglected the induced polarization and the difference between the external electric field and the internal electric field acting on a molecule. The susceptibilities depend on the density, square of the dipolar moment, temperature, and the order parameters. The anisotropy $\Delta \chi=\chi_{z z}-\chi_{x x}$ is positive as a result of the assumption on the dipole moment orientation.

Our calculations show that for positive or small negative values of $v_{1}$ the ordering is the same as in the case of $v_{1}=0$ (uniaxial molecules). Below the isotropic phase there is the nematic phase with the $D_{\infty h}$ symmetry. On the other hand, the nonzero $v_{1}$ strongly changes the component of the susceptibility which is parallel to the symmetry axis of the molecule. We also find that for $v_{1}>0$ the average susceptibility is lower than the isotropic susceptibility which is in agreement with the experiments for polar molecules.

The presented model describes only liquid crystal phases with translational symmetry. For real liquid crystals below the nematic phase (for lower temperatures) one can usually find the smectic phase translationally ordered in one direction (rod-like molecules) or the columnar phase translationally ordered in two directions (disc-like molecules) [14]. Sometimes the nematic phase is even absent. It is possible to extend our model for smectic phases by extension of the McMillan model [15] or to include the columnar phases by extension of the model by Feldkamp et al. [16]. Work is in progress on the theory where many limitations of the presented approach is removed, i.e. any orientation of the dipole moment 
is possible, the induced polarization and difference between external and internal electric fields are taken into account.

\section{Appendix A}

Below we list the properties of the functions $E_{\mu \nu}^{(j)}$. The functions can be used to describe any physical quantity which depends on the three Euler angles.

1. The definition is

$$
\begin{aligned}
& E_{\mu \nu}^{(j)}(R)=\left(\frac{1}{\sqrt{2}}\right)^{2+\delta_{0 \mu}+\delta_{0 \nu}} \frac{1}{2}[(1+\mathrm{i})+(1-\mathrm{i}) \operatorname{sign}(\mu) \operatorname{sign}(\nu)] \\
& \times {\left[D_{\mu \nu}^{(j)}(R)+\operatorname{sign}(\mu) \operatorname{sign}(\nu)(-1)^{\mu+\nu} D_{-\mu,-\nu}^{(j)}(R)\right.} \\
&\left.\quad+\operatorname{sign}(\nu)(-1)^{\nu} D_{\mu,-\nu}^{(j)}(R)+\operatorname{sign}(\mu)(-1)^{\mu} D_{-\mu, \nu}^{(j)}(R)\right],
\end{aligned}
$$

where $R=(\phi, \theta, \psi)$ (the three Euler angles), $j$ is a non-negative integer, $\mu$ and $\nu$ are integers. Functions $D_{\mu \nu}^{(j)}$ are standard rotation matrix elements [17] and

$$
\operatorname{sign}(x)=\left\{\begin{aligned}
1 & \text { for } x \geq 0, \\
-1 & \text { for } x<0 .
\end{aligned}\right.
$$

Let us note that

$$
\operatorname{sign}(-x)=-\operatorname{sign}(x)+2 \delta_{0 x} .
$$

2. The functions $E_{\mu \nu}^{(j)}$ are real.

3. The functions satisfy the orthogonality relations

$$
\int \mathrm{d} R E_{\mu \nu}^{(j)}(R) E_{\rho \sigma}^{(k)}(R)=\delta_{j k} \delta_{\mu \rho} \delta_{\nu \sigma} 8 \pi^{2} /(2 j+1) .
$$

4. Let us assume that the three Euler angles $R=(\phi, \theta, \psi)$ determine the orientation of the three unit orthogonal vectors $(\boldsymbol{l}, \boldsymbol{m}, \boldsymbol{n})$. The coordinates of the vectors can be expressed by means of the functions $E_{\mu \nu}^{(1)}$ :

$$
\begin{aligned}
& l_{x}=E_{11}^{(1)}(R)=\cos \theta \cos \phi \cos \psi-\sin \phi \sin \psi, \\
& l_{y}=-E_{-11}^{(1)}(R)=\cos \theta \sin \phi \cos \psi+\cos \phi \sin \psi, \\
& l_{z}=E_{01}^{(1)}(R)=-\sin \theta \cos \psi, \\
& m_{x}=E_{1-1}^{(1)}(R)=-\cos \theta \cos \phi \sin \psi-\sin \phi \cos \psi, \\
& m_{y}=E_{-1-1}^{(1)}(R)=-\cos \theta \sin \phi \sin \psi+\cos \phi \cos \psi, \\
& m_{z}=E_{0-1}^{(1)}(R)=\sin \theta \sin \psi, \\
& n_{x}=E_{10}^{(1)}(R)=\sin \theta \cos \phi, \\
& n_{y}=-E_{-10}^{(1)}(R)=\sin \theta \sin \phi,
\end{aligned}
$$


$n_{z}=E_{00}^{(1)}(R)=\cos \theta$.

5. The functions $E_{\mu 0}^{(j)}$ are proper for the description of uniaxial molecules. We can express them as products $n_{x}^{a} n_{y}^{b} n_{z}^{c}$, where $b=0$ or $b=1$.

$$
\begin{aligned}
& E_{00}^{(0)}=1, \\
& E_{10}^{(1)}=n_{x}, \\
& E_{00}^{(1)}=n_{z}, \\
& E_{-10}^{(1)}=-n_{y}, \\
& E_{20}^{(2)}=(1 / 2)\left(-1+n_{z}^{2}+2 n_{x}^{2}\right) \sqrt{3}, \\
& E_{10}^{(2)}=n_{x} n_{z} \sqrt{3}, \\
& E_{00}^{(2)}=-1 / 2+(3 / 2) n_{z}^{2}, \\
& E_{-10}^{(2)}=-n_{z} n_{y} \sqrt{3}, \\
& E_{-20}^{(2)}=n_{x} n_{y} \sqrt{3}, \\
& E_{30}^{(3)}=(1 / 4) n_{x}\left(-3+3 n_{z}^{2}+4 n_{x}^{2}\right) \sqrt{5} \sqrt{2}, \\
& E_{20}^{(3)}=(1 / 2) n_{z}\left(-1+n_{z}^{2}+2 n_{x}^{2}\right) \sqrt{3} \sqrt{5}, \\
& E_{10}^{(3)}=(1 / 4) n_{x}\left(-1+5 n_{z}^{2}\right) \sqrt{3} \sqrt{2}, \\
& E_{00}^{(3)}=(-3 / 2) n_{z}+(5 / 2) n_{z}^{3}, \\
& E_{-10}^{(3)}=(-1 / 4) n_{y}\left(-1+5 n_{z}^{2}\right) \sqrt{3} \sqrt{2}, \\
& E_{-20}^{(3)}=n_{x} n_{z} n_{y} \sqrt{3} \sqrt{5}, \\
& E_{-30}^{(3)}=(-1 / 4)\left(-1+n_{z}^{2}+4 n_{x}^{2}\right) n_{y} \sqrt{5} \sqrt{2}, \\
& E_{40}^{(4)}=(1 / 8)\left(1-2 n_{z}^{2}-8 n_{x}^{2}+n_{z}^{4}+8 n_{x}^{2} n_{z}^{2}+8 n_{x}^{4}\right) \sqrt{35}, \\
& E_{30}^{(4)}=(1 / 4) n_{x} n_{z}\left(-3+3 n_{z}^{2}+4 n_{x}^{2}\right) \sqrt{35} \sqrt{2}, \\
& E_{20}^{(4)}=(1 / 4)\left(1-8 n_{z}^{2}-2 n_{x}^{2}+7 n_{z}^{4}+14 n_{x}^{2} n_{z}^{2}\right) \sqrt{5}, \\
& E_{10}^{(4)}=(1 / 4) n_{x} n_{z}\left(-3+7 n_{z}^{2}\right) \sqrt{5} \sqrt{2}, \\
& E_{00}^{(4)}=3 / 8-(15 / 4) n_{z}^{2}+(35 / 8) n_{z}^{4},
\end{aligned}
$$




$$
\begin{aligned}
& E_{-10}^{(4)}=(-1 / 4) n_{z} n_{y}\left(-3+7 n_{z}^{2}\right) \sqrt{5} \sqrt{2}, \\
& E_{-20}^{(4)}=(1 / 2) n_{x} n_{y}\left(-1+7 n_{z}^{2}\right) \sqrt{5} \\
& E_{-30}^{(4)}=(-1 / 4) n_{z} n_{y}\left(-1+n_{z}^{2}+4 n_{x}^{2}\right) \sqrt{35} \sqrt{2}, \\
& E_{-40}^{(4)}=(1 / 2) n_{x} n_{y}\left(-1+n_{z}^{2}+2 n_{x}^{2}\right) \sqrt{35} .
\end{aligned}
$$

\section{Appendix B}

Excluded volumes $V_{\text {excl }}$ for uniaxial molecules with the $D_{\infty h}$ and $C_{\infty v}$ symmetry. $V_{\text {mol }}$ denotes the volume of a molecule. The excluded volumes were used to calculate the parameters of the interactions $v_{j}(j=0,1,2)$ from the expressions

$V_{\text {excl }}\left(\boldsymbol{e}_{z}, \boldsymbol{e}_{z}\right)$ is proportional to $v_{0}+v_{1}+v_{2}$,

$V_{\text {excl }}\left(\boldsymbol{e}_{z},-\boldsymbol{e}_{z}\right)$ is proportional to $v_{0}-v_{1}+v_{2}$,

$V_{\text {excl }}\left(\boldsymbol{e}_{z}, \boldsymbol{e}_{x}\right)$ is proportional to $v_{0}-v_{2} / 2$.

1. Ellipsoids with the axes $2 a \times 2 a \times 2 c$ ( $D_{\infty h}$ symmetry).

$V_{\mathrm{mol}}=(4 / 3) \pi a^{2} c$

$V_{\mathrm{excl}}\left(\boldsymbol{e}_{z}, \boldsymbol{e}_{z}\right)=8 V_{\mathrm{mol}}$

$V_{\mathrm{excl}}\left(\boldsymbol{e}_{z}, \boldsymbol{e}_{x}\right) \approx(4 / 3) \pi(a+c)^{2}(2 a)$.

2. Cylinders with the length $H$ and the diameter $2 R$ ( $D_{\infty h}$ symmetry).

$V_{\mathrm{mol}}=\pi R^{2} H$

$V_{\mathrm{excl}}\left(\boldsymbol{e}_{z}, \boldsymbol{e}_{z}\right)=8 V_{\mathrm{mol}}$

$V_{\mathrm{excl}}\left(\boldsymbol{e}_{z}, \boldsymbol{e}_{x}\right)=4 \pi R^{3}+2 \pi R^{2} H+4 R H^{2}$.

3. Cones with the height $H$ and the diameter of the base $2 R\left(C_{\infty v}\right.$ symmetry).

$$
\begin{aligned}
& V_{\mathrm{mol}}=(1 / 3) \pi R^{2} H, \\
& V_{\mathrm{excl}}\left(\boldsymbol{e}_{z}, \boldsymbol{e}_{z}\right)=14 V_{\mathrm{mol}}, \\
& V_{\mathrm{excl}}\left(\boldsymbol{e}_{z},-\boldsymbol{e}_{z}\right)=8 V_{\mathrm{mol}}, \\
& V_{\mathrm{excl}}\left(\boldsymbol{e}_{z}, \boldsymbol{e}_{x}\right) \approx 12 R^{3}+8 R^{2} H+4 R H^{2} .
\end{aligned}
$$

4. "Towers" that consist of two cylinders which have the common axis of rotational symmetry $\left(C_{\infty v}\right.$ symmetry). The cylinders are of the height $H / 2$ and the diameters $R$ and $2 R$. 


$$
\begin{aligned}
& V_{\mathrm{mol}}=(5 / 8) \pi R^{2} H, \\
& V_{\mathrm{excl}}\left(\boldsymbol{e}_{z}, \boldsymbol{e}_{z}\right)=10 V_{\mathrm{mol}}, \\
& V_{\mathrm{excl}}\left(\boldsymbol{e}_{z},-\boldsymbol{e}_{z}\right)=9 V_{\mathrm{mol}}, \\
& V_{\mathrm{excl}}\left(\boldsymbol{e}_{z}, \boldsymbol{e}_{x}\right) \approx 15 R^{3}+12 R^{2} H+4 R H^{2} .
\end{aligned}
$$

\section{References}

[1] P.G. de Gennes, J. Prost, The Physics of Liquid Crystals, Clarendon Press, Oxford 1993.

[2] E. Garcia, D.C. Williamson, A. Martinez-Richa, Mol. Phys. 98, 179 (2002).

[3] A.N. Zakhlevnykh, P.A. Sosnin, Mol. Cryst. Liq. Cryst. 293, 135 (1997).

[4] B. Tjipto-Margo, G.T. Evans, M.P. Allen, D. Frenkel, J. Phys. Chem. 96, 3942 (1992).

[5] Z. Zhang, M.J. Zuckermann, O.G. Mouritsen, Mol. Phys. 80, 1195 (1993).

[6] A.M. Somoza, P. Tarazona, Mol. Phys. 75, 17 (1992).

[7] F. Barmes, M. Ricci, C. Zannoni, D.J. Cleaver, Phys. Rev. E 68, 021708 (2003).

[8] A.J. Davidson, N.J. Mottram, Phys. Rev. E 65, 051710 (2002).

[9] J.P. Straley, Phys. Rev. A 10, 1881 (1974).

[10] W. Maier, G.Z. Meier, Z. Naturforsch. A 16, 262, 470, 1200 (1961).

[11] W.H. de Jeu, Solid State Physics, Supplement 14, Liquid Crystals, Academic Press, New York 1978.

[12] R.B. Meyer, Phys. Rev. Lett. 22, 918 (1969).

[13] S.T. Lagerwall, in: Handbook of Liquid Crystals, Eds. D. Demus, J. Goodby, G.W. Gray, H.-W. Spiess, V. Vill, Vol. 2B, Wiley-VCH, Weinheim 1998.

[14] S. Chandrasekhar, G.S. Ranganath, Rep. Prog. Phys. 53, 57 (1990).

[15] W.L. McMillan, Phys. Rev. A 4, 1238 (1971).

[16] G.E. Feldkamp, M.A. Handschy, N.A. Clark, Phys. Lett. A 85, 359 (1981).

[17] A.R. Edmonds, Angular Momentum in Quantum Mechanics, Princeton U.P., Princeton, N.Y. 1957. 